\title{
On the two-dimensional stability of the axisymmetric Burgers vortex
}

\author{
A. Prochazka and D. J. Pullin \\ Graduate Aeronautical Laboratories 205-45, California Institute of Technology, Pasadena, California 91125
}

(Received 28 December 1994; accepted 16 February 1995)

The stability of the axisymmetric Burgers vortex solution of the Navicr-Stokes equations to two-dimensional perturbations is studied numerically up to Reynolds numbers, $R=\Gamma / 2 \pi \nu$, of order $10^{4}$. No unstable eigenmodes for azimuthal mode numbers $n=1, \ldots, 10$ are found in this range of Reynolds numbers. Increasing the Reynolds number has a stabilizing effect on the vortex. (c) 1995 American Institute of Physics.

Taylor $^{1}$ was the first to note the importance of the interaction of vortex stretching and viscosity to intensify and diffuse vorticity in the process of dissipating energy in turbulence. A solution to the Navier-Stokes equations that models this interaction was discovered by Burgers. ${ }^{2}$ This has been used in turbulence applications by Townsend ${ }^{3}$ and others. Large-scale numerical simulations by She, Jackson, and Orszag, ${ }^{4}$ Vincent and Meneguzzi, ${ }^{5}$ and Jiménez et al. ${ }^{6}$ have indicated the presence of vortices whose characteristics resemble those of Burgers vortices. The stability of the Burgers vortex in both two and three dimensions is therefore of interest. This problem was studied analytically for low Reynolds numbers by Robinson and Saffman ${ }^{7}$ henceforth referred to as RS.

The problem will be formulated in the cylindrical polar coordinate system $(r, \theta, z)$ where the velocity field in the respective directions will be denoted by $\mathbf{u}=\left(u_{r}, u_{\theta}, u_{z}\right)$. This velocity field is decomposed as

$$
\begin{aligned}
& u_{r}=-(\gamma / 2) r+\hat{u}_{r}(r, \theta, t), \\
& u_{\theta}=\hat{u}_{\theta}(r, \theta, t), \\
& u_{z}=\gamma z,
\end{aligned}
$$

where $\hat{u}_{r}$ and $\hat{u}_{\theta}$ are the rotational part of the velocity field and $\gamma$ is the rate of strain in the $z$ direction corresponding to a uniform axisymmetric external strain. Substitution of (1)(3) into the vorticity equation gives

$\frac{\partial \omega}{\partial t}+u_{r} \frac{\partial \omega}{\partial r}+\frac{u_{\theta}}{r} \frac{\partial \omega}{\partial \theta}=\gamma \omega+\frac{\nu}{r}\left[\frac{\partial}{\partial r}\left(r \frac{\partial \omega}{\partial r}\right)+\frac{1}{r} \frac{\partial^{2} \omega}{\partial \theta^{2}}\right]$,

where the vorticity field is nonzero only in the $z$ direction $\omega=(0,0, \omega)$. It is well known that a steady axisymmetric solution of (4) is given by

$$
\omega_{0}(r)=(\gamma \Gamma / 4 \pi \nu) e^{-\gamma r^{2} / 4 \nu},
$$

which induces the angular velocity,

$$
\hat{u}_{\theta 0}=(\Gamma / 2 \pi r)\left(1-e^{-\gamma r^{2} / 4 \nu}\right),
$$

where $\Gamma$ is the total circulation. Equations $(5)-(6)$ are the axisymmetric Burgers vortex and represent an equilibrium which is a balance between the stretching of vortex lines by the external strain and the lateral diffusion of vorlicity.

While the stability of (5) -(6) to three-dimensional (3-D) perturbations is of fundamental interest, we presently consider the stability to two-dimensional (2-D) disturbances. All quantities are nondimensionalized with respect to length scale $\sqrt{\nu / \gamma}$ and time scale $\gamma^{-1}$. The 2-D perturbed velocity field in the $r-\theta$ plane is

$$
\begin{aligned}
& u_{r}=u_{r 0}+u_{r}^{\prime}(r, \theta, t), \quad u_{\theta}=u_{\theta 0}+u_{\theta}^{\prime}(r, \theta, t), \\
& \omega=\omega_{0}+\omega^{\prime}(r, \theta, t) .
\end{aligned}
$$

A perturbation streamfunction $\psi^{\prime}$ can be defined such that

$$
\begin{aligned}
& u_{r}^{\prime}=\frac{1}{r} \frac{\partial \psi^{\prime}}{\partial \theta}, \quad u_{\theta}^{\prime}=-\frac{\partial \psi^{\prime}}{\partial r}, \\
& \nabla^{2} \psi^{\prime}=-\omega^{\prime} .
\end{aligned}
$$

The perturbations are assumed to be of the normal mode form

$$
\begin{aligned}
& \omega^{\prime}=\omega(r) e^{-\mu t+i n \theta}, \\
& \psi^{\prime}=\psi(r) e^{-\mu t+i n \theta},
\end{aligned}
$$

where $n$ is the azimuthal mode and $-\mu$ is the complex growth rate of the perturbation. In this formulation, unstable solutions correspond to negative values of the real part of $\mu$. Using (8)-(11) in (4) and linearizing, it is found that

$$
\begin{aligned}
& L^{n} \omega+\mu \omega=\operatorname{in} R\left(-f \psi+\frac{1}{2} g \omega\right), \\
& M^{n} \psi=-\omega,
\end{aligned}
$$

where $R=\Gamma / 2 \pi \nu$ is a Reynolds number, the primes on the perturbation variables have been dropped for convenience, and

$$
\begin{aligned}
& M^{n}(\cdot) \equiv \frac{1}{r} \frac{d}{d r}\left(r \frac{d}{d r}(\cdot)\right)-\frac{n^{2}}{r^{2}}(\cdot), \\
& L^{n}(\cdot) \equiv M^{n}(\cdot)+\frac{1}{r} \frac{d}{d r}\left[r^{2}(\cdot)\right], \\
& f \equiv e^{-r^{2} / 2}, \quad g \equiv\left(1-e^{-r^{2} / 2}\right) /\left(r^{2} / 2\right) .
\end{aligned}
$$

The first part of the RS analysis dealt with the special case of $R=0$; the Stokes limit of (1)-(4) with the present scaling. Solutions were found in terms of the confluent hypergeometric function and, enforcing exponential decay at infinity, gave eigenvalues $\mu=n+2 k, k=0,1,2, \ldots$. Since the eigenvalues are all positive real, the Burgers vortex is stable for zero circulation. The $R=0$ normalized vorticity eigenfunctions are 


$$
\omega(n, k)=2^{-n / 2} r^{n} e^{-r^{2} / 2} L_{k}^{(n)}\left(r^{2} / 2\right) .
$$

The streamfunction eigenfunctions can be found by integrating the vorticity eigenfunctions to give

$\psi(r ; n, 0)=\frac{1}{2}\left[\Gamma(n) /\left(r^{2} / 2\right)^{n / 2}\right]\left[1-e_{n-1}\left(r^{2} / 2\right) e^{-r^{2} / 2}\right]$,

$\psi(r ; n, k)=(1 / 2 k) r^{n} e^{=r^{2} / 2} L_{k-1}^{(n)}\left(r^{2} / 2\right)$,

where $e_{n-1}$ is the exponential serics truncated after $n-1$ terms.

In order to tackle the problem numerically, we first obtain from (12)-(13) one equation for the vorticity,

$$
g(\omega)=-\mu \omega
$$

which is a complex eigenvalue problem with

$$
\mathscr{E}(\cdot)=L^{n}(\cdot)-i n R\left[f\left(M^{n}\right)^{-1}(\cdot)+\frac{1}{2} g(\cdot)\right] \text {. }
$$

The bchavior at the boundaries for the finite Reynolds number problem is identical to that found for the $R=0$ case. Using boundedness of solutions and a Frobenius method about the regular singular point, $r=0$, we find the behavior of the vorticity to be,

$$
\omega=O\left(r^{n}\right), \quad r \rightarrow 0 .
$$

For $r \rightarrow \infty$, a dominant balance argument is used to write Eq. (20) as

$$
\frac{1}{r} \frac{d}{d r}\left(r \frac{d}{d r} \omega\right)-\frac{n^{2}}{r^{2}} \omega+\frac{1}{r} \frac{d}{d r}\left[r^{2} \omega\right]+\mu \omega=\frac{i n R \omega}{r^{2}},
$$

which can be written simply as

$$
L^{\left(n^{2}+i n R\right)^{1 / 2}}(\omega)+\mu \omega=0,
$$

so that the behavior at infinity remains the same as for $R=0$,

$$
\omega \sim A r^{\mu} e^{-r^{2} / 2}, \quad r \rightarrow \infty
$$

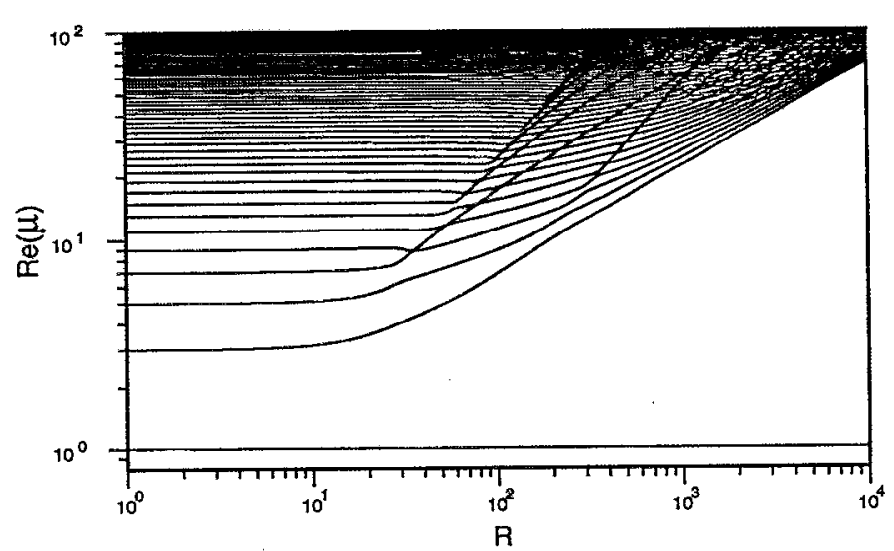

FIG. 1. Real part of the eigenvalues versus Reynolds number for $n=1$, $N=300, k=0,1,2, \ldots$ defined by the $y$ axis intercept, $n+2 k$.

A Galerkin approach was used to expand solutions in the orthogonal basis defined by the $R=0$ vorticity eigenfunctions

$\omega=\sum_{k=0}^{N-1} a_{k} \omega(r ; n, k), \quad \omega(r ; n, k)=2^{-n / 2} r^{n} e^{-r^{2} / 2} L_{k}^{(n)}\left(\frac{r^{2}}{2}\right)$

where the finite expansion is truncated at $N-1$ terms. Substitution of (26) into (20) results in a complex eigenvalue problem of the form

$$
\mathbf{A x}=-\mu \mathbf{x}
$$

where $\mathbf{x}=\left(a_{0}, a_{1}, \ldots, a_{N-1}\right)^{T}$ and $\mathbf{A}$ is complex matrix of or$\operatorname{der} N \times N$ whose coefficients are found by using the orthogonality of the basis functions under the weighted inner product,

TABLE I. Comparison with Robinson and Saffman's results for small Reynolds number, $N=50$, and $\mu=\mu_{0}+R \mu_{1}+R^{2} \mu_{2}$.

\begin{tabular}{cclccc}
\hline$n$ & $k$ & $-i \mu_{1}(\mathrm{RS})$ & $-i \mu_{1}$ & $10^{2} \mu_{2}(\mathrm{RS})$ & $10^{2} \mu_{2}$ \\
\hline 1 & 0 & 0 & 0.00000000 & 0 & 0.00000000 \\
1 & 1 & 0.125 & 0.12499977 & 0.14435071 & 0.14435055 \\
1 & 2 & 0.125 & 0.12500003 & 0.10094794 & 0.10094833 \\
$\vdots$ & $\vdots$ & $\vdots$ & $\vdots$ & $\vdots$ & $\vdots$ \\
1 & 7 & 0.0916443 & 0.09164255 & 0.00225961 & 0.00225988 \\
1 & 8 & 0.0872803 & 0.08728127 & -0.000431672 & -0.00043345 \\
1 & 9 & 0.0834618 & 0.08346671 & -0.00207814 & -0.00207772 \\
2 & 0 & 0.025 & 0.02499994 & 0.27855829 & 0.27855588 \\
2 & 1 & 0.025 & 0.02499994 & 0.42798057 & 0.42798354 \\
2 & 2 & 0.0234375 & 0.02343752 & 0.02636197 & 0.02636217 \\
$\vdots$ & $\vdots$ & $\vdots$ & $\vdots$ & $\vdots$ & $\vdots$ \\
2 & 8 & 0.166924 & 0.16692388 & 0.00340799 & 0.00310921 \\
2 & 9 & 0.160179 & 0.16017876 & -0.00284228 & -0.00284910 \\
2 & 10 & 0.154172 & 0.15417211 & -0.00697667 & -0.00697291 \\
3 & 0 & 0.0375 & 0.03749983 & 0.85088957 & 0.85088854 \\
3 & 1 & 0.034375 & 0.03437489 & 0.64678972 & 0.64679239 \\
3 & 2 & 0.03203125 & 0.03203126 & 0.43069801 & $\vdots$ \\
$\vdots$ & $\vdots$ & $\vdots$ & $\vdots$ & 0.43070205 \\
3 & 10 & 0.220477 & 0.22048121 & 0.00131356 & $\vdots$ \\
3 & 11 & 0.213431 & 0.21342685 & -0.00600394 & -0.00601022 \\
3 & 12 & 0.20701 & 0.20722134 & -0.0112759 & -0.01131203 \\
\hline
\end{tabular}




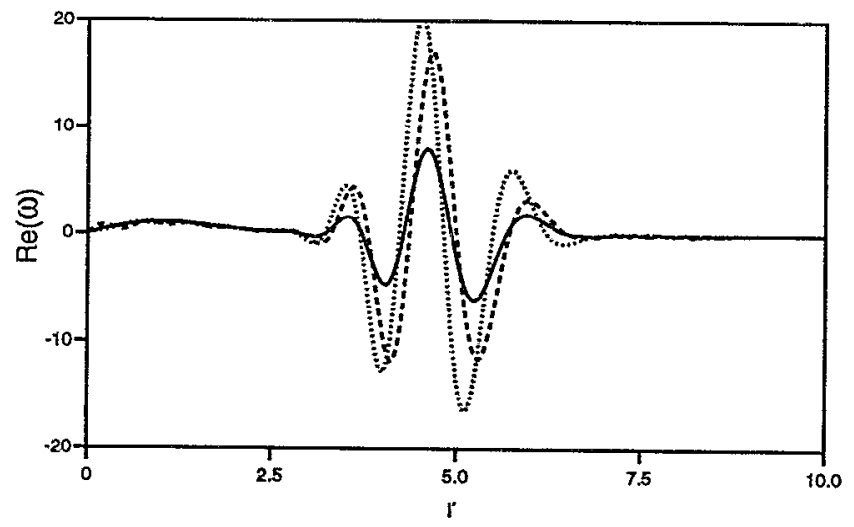

FIG. 2. Real part of the eigenfunctions versus radius for $n=1, N=300$, $R=1000, k=1$ (solid), 2 (dashed), 3 (dotted).

$$
\langle u, v\rangle=\int_{0}^{\infty} u \bar{v} e^{r^{2} / 2} r d r
$$

such that the coefficients of $\mathbf{A}$,

$$
\mathbf{A}_{j k}=\left\langle\omega_{j}^{n}(r), \mathscr{L} \omega_{k}^{n}(r)\right\rangle /\left\langle\omega_{j}^{n}(r), \omega_{j}^{n}(r)\right\rangle
$$

can be easily calculated.

By construction, this method reproduces the known eigensolutions for $R=0$. Results for larger Reynolds number are plotted in Fig. 1 for $n=1$. Numerical solutions to (27) were found using the $\mathrm{QZ}$ algorithm for values of $N$ ranging from 20 up to 300 . For fixed $N$ and $n$, we find a denumerable set of eigenfunctions and eigenvalues for $k=0,1,2, \ldots$, each corresponding to a different radial structure of the perturbation. These calculations were tested in various ways. First, for low Reynolds number, our results were compared to and agreed well with the RS results (see Table I and subsequent discussion). Next, for fixed $R$, the dimension $N$ was increased until convergence was evident. Third, results for Reynolds numbers up to approximately 100 were checked by treating the problem as a nonlinear continuation in the parameter $R$, starting at $R=0$, using a Newton-Raphson scheme with finite-difference techniques.

RS did analyze the effect of nonzero Reynolds number by way of a perturbation expansion for small $R$ and found that for the eigenvalue expansion

$$
\mu=\mu_{0}+\mu_{1} R+\mu_{2} R^{2}+\cdots,
$$

$\mu_{0}=n+2 k$ and the coefficients $\mu_{1}$ and $\mu_{2}$ were given by RS:\{(2.15)-(2.19)\}. Since $\mu_{1}$ is strictly imaginary its value does not affect the stability of the vortex. For low values of $k, \mu_{2}$ is positive and thereby serves only to increase the value of $\mathfrak{R}(\mu)$. We have calculated $\mu_{1}$ and $\mu_{2}$ to larger $k$ than RS, and find that, for fixed $n, \mu_{2}$ can become negative at sufficiently large $k$ (see Table I). This is equivalent to $\left(\partial^{2} \mu / \partial R^{2}\right)_{R=0}<0$ and indicates a trend toward possible instability at values of $R$ beyond the validity of (30).

Values of $n=1, \ldots, 10$ were calculated. In all cases, as illustrated in Fig. 1, $\operatorname{Re}(\mu)>0$, indicating stability of the Burgers vortex to 2-D perturbations. Note that for values of $(n, k)$, where (30) indicates $\left(\partial^{2} \mu / \partial R^{2}\right)_{R=0}<0$, we found that $\mathfrak{R}(\mu)$ reached a local minimum followed by a subsequent increase with increasing $R$. This behavior is on a scale too small to be seen graphically in Fig. 1. Numerical results at high Reynolds indicate that the real part of the eigenvalue grows like the square root of the Reynolds number. The irregularity of the plots at large Reynolds number is caused by resolution problems in representing the vorticity with a maximum number of basis functions, $N=300$.

It was found that, as $R$ was increased at fixed $n, k$, the eigenfunction became increasingly oscillatory, whilst remaining confined to within a narrow band. This is illustrated in Fig. 2, which shows the real part of the eigenfunction for $n=1, R=1000$. It was this structure that limited the range of our finite-difference method to maximum $R \sim 10^{2}$ and that of the spectral method to maximum $R \sim 10^{4}$.

\section{ACKNOWLEDGMENTS}

Helpful discussions with P. G. Saffman and D. Crowdy are gratefully acknowledged. This research was partial supported by National Science Foundation Grant No. CTS9311811.

${ }^{1}$ G. I. Taylor, Proc. R. Soc. London Ser. A 164, 15 (1938).

${ }^{2}$ J. M. Burgers, Adv. Appl. Mech. 1, 171 (1948).

${ }^{3}$ A. A. Townsend, Proc. R. Soc. London Ser. A 208, 534 (1938).

${ }^{4}$ Z. S. She, E. Jackson, and S. A. Orszag, Proc. R. Soc. London Ser. A 434, 101 (1991).

${ }^{5}$ A. Vincent and M. Meneguzzi, J. Fluid Mech. 225, 1 (1991)

${ }^{6}$ J. Jiménez, A. A. Wray, P. Saffman, and R. S. Rogallo, J. Fluid Mech. 255, 65 (1993).

${ }^{7}$ A. C. Robinson and P. G. Saffman, Stud. Appl. Math. 70, 163 (1984). 